\title{
Apreciación folclórica del género musical en la provincia de Sihuas Ancash, 2016
}

Folk appreciation of the musical genre in the province of Sihuas Ancash, 2016

Fortunato Diestra Salinas ${ }^{1}$

\section{RESUMEN}

El objetivo de la investigación es determinar el grado de apreciación folclórica en el género musical de los pobladores de la provincia de Sihuas, Ancash 2016.Se realizó utilizando el método científico. La investigación es de tipo básico, diseño de investigación descriptivo, mediante el cual se recoge la información por primera vez en el lugar de nuestro estudio , para ello se utilizó como instrumento un cuestionario elaborado en la escala de Likert con 12 preguntas, previamente fue validado por los expertos tanto el constructo como la fiabilidad, posteriormente se procedió a recoger la información, para luego procesar los datos, haciendo uso de la estadística descriptiva, teniendo en cuenta los cuatro géneros musicales que se practican en la provincia, el género musical chuscada, la música chacrita, el chimaychi y el género pasacalle. Obteniendo como resultados que La chuscada goza de mayor preferencia con un $82 \%$ de preferencia ubicándose en el primer lugar, seguido por la música chacrita con un $47 \%$ de preferencia. El chimaychi goza de una preferencia de $37 \%$ ubicándose en el tercer lugar. El pasacalle goza de una preferencia de $17 \%$ ubicándose en el cuarto lugar de preferencia. Llegando a la conclusión, que se ha logrado conocer cuál es la apreciación folclórica musical en la provincia de Sihuas departamento Ancash. 2016. De esta manera, nos ayudará tener en cuenta la preferencia y/o la apreciación del género musical, sirviendo para profundizar nuestra investigación y obtener otros resultados de interés provincial.

\section{Palabras clave: Apreciación folclórica musical.}

\section{ABSTRACT}

The objective of the research is to determine the degree of appreciation of folk in the musical genre of the inhabitants of the province of Sihuas Ancash 2016.Se performed using the scientific method. The research is basic, descriptive research design, which contains information for the first time in the place of our study, was used as an instrument a questionnaire developed in the Likert scale with 1 2questions, was previously validated by experts both reliability and construct, then proceeded to collect information, to then process the data, making use of descriptive statistics, taking into account the four musical genres that are they practice in the province, the musical genre hortatory, music lands, the chimaychi and the genu sparade. Obtaining results which the hortatory enjoys greater preference with $82 \%$ of preference ranking in first place, followed by the music lands with a $47 \%$ preference. The chimaychi has a preference of $37 \%$ rank in third place. The parade has a preference of $17 \%$ in the fourth place of preference Coming to the conclusion, that have been know what folk music appreciation in the province of Sihuas Ancash Department.2016. in this way it will help us to take into account the preference or the appreciation of the genre, serving to deepen our research and other results of provincial interest

Keywords: Folk music appreciation. 


\section{INTRODUCCIÓN}

Nuestra inquietud de realizar la presente investigación, nace como resultado de dar solución a una problemática de suma importancia en la provincia de Sihuas, ¿cuál es?, conocer las preferencias o apreciación del poblador, referente al folclor especialmente en la música. Sihuas, uno de las provincias del departamento de Ancash, no puede ser ajeno a ello, por ser un pueblo forjado dentro del ambiente musical, que se viene practicando en sus diferentes distritos y pueblos que conforman la provincia. Desde la época de su origen, conocida a través de la historia, con su paso por la época preincaica floreció la cultura Chavín, de la cual las manifestaciones folclóricas, entre ellas la música. Posteriormente con la presencia de la cultura incaica, y la invasión española que le aportaron cada uno de ellas lo referente al folclor como la música considerada como el arte para combinar los sonidos y teniendo en cuenta las leyes de la armonía, melodía y el ritmo que se producirán al ejecutar los instrumentos musicales.

Considerando que el folclor de los pueblos son manifestaciones de la sabiduría popular, se pretende conocer cómo se valora y aprecia esta riqueza cultural; además, de las posibilidades de tener un material de inicio de una investigación que puede servir de antecedente o guía a los futuros investigadores en este tema.

Muchos estudiosos consideran al "huayno" de origen andino, aparecido en época prehispánica, como procedente de los quechuas y aymaras, y que posteriormente con la formación del imperio de los incas se ha expandido por todas las regiones y pueblos que pertenecían al imperio. Otros sostienen que el nombre de huayno proviene de una voz quechua "huayñunakunay" que significa bailar tomados de la mano. Estas consideraciones son importantes en nuestro estudio en el departamento de Ancash provincia de Sihuas y, entender cómo entiende el concepto de huayno el poblador.

¿Cuál es la apreciación folclórica del género musical en la provincia de Sihuas departamento Ancash. 2016?

El objetivo de este estudio es conocer cuál es el grado de apreciación folclórica del género musical en la provincia de Sihuas departamento Ancash, 2016.

\section{MATERIAL Y MÉTODOS}

La investigación es básica, de nivel descriptivo simple, porque se trabajó con una sola variable, que solo permitió recoger la información y explicar del fenómeno observado mediante la descripción, argumentando todo el proceso tal conforme se observó y se produjeron los hechos interpretándolos de acuerdo al resultado.

La muestra con lo que se trabajó fue de 60 personas seleccionados mediante muestras no probabilísticas.

Se ha tomado una parte o fragmento de la población con los cuales se ha realizado todo el proceso de la investigación, mediante la información proporcionada por ellos.

El instrumento que se utilizó para la recopilación de datos, fue el cuestionario elaborado en la escala de Likert, con un contenido de 12 preguntas referente a la apreciación que tiene el poblador de las manifestaciones musicales existentes de la provincia de Sihuas. Previamente el constructo del instrumento fue elaborado teniendo en cuenta las teorías que se han utilizado en el marco teórico, con la finalidad de darle mayor validez al instrumento. Posteriormente validado por juicio de expertos, con grados académicos de magister y doctor, después de sus observaciones, y haber subsanado, se entrevistó a quince personas de la muestra para recoger la información, con los que se hizo la prueba de la fiabilidad utilizando el estadístico alfa de Cronbach.

Tabla 1. Estadísticos de fiabilidad.

\begin{tabular}{ll}
\hline Alfa de Cronbach & $\mathbf{N}$ de elementos \\
\hline 0,874 & 15 \\
\hline
\end{tabular}

El coeficiente alfa de Cronbach da un resultado de alta confiabilidad, siendo de 0,874 que se aproxima a 1. Después de todo ello se aplicó el cuestionario a la muestra, recogiendo los datos requeridos, los cuales fueron procesados ordenados para la interpretación, tal conforme se demuestra en los siguientes cuadros. 


\section{RESULTADOS}

Tabla 2. Frecuencia y porcentaje de preciación a la chuscada por el poblador.

\begin{tabular}{clrc}
\hline & & Frecuencia & Porcentaje \\
\hline Válido & Poco & 5 & $8 \%$ \\
& Regular & 6 & $10 \%$ \\
& Bastante & 49 & $82 \%$ \\
& Total & 60 & $100 \%$ \\
\hline
\end{tabular}

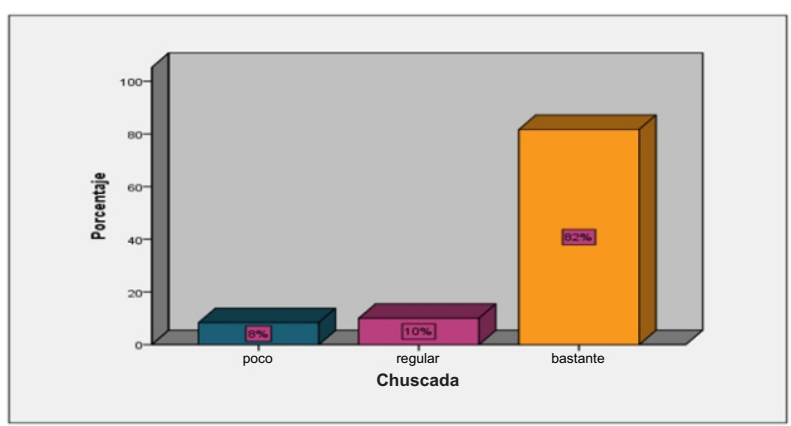

Figura 1. Gráfico de apreciación a la chuscada por el poblador.

En la Tabla 1 y Figura 1 se observa que 5 encuestados que representa el $8 \%$, manifiestan que, tienen poco apreciación a la chuscada, de la misma manera 6 de los encuestados que representan el $10 \%$ manifiestan que tienen apreciación regular, y 49 de los encuestados que representa el $82 \%$ manifiestan que su apreciación es bastante.

Tabla 3. Frecuencia y porcentaje de apreciación de la música chacrita por el poblador.

\begin{tabular}{clc|c}
\hline & & Frecuencia & Porcentaje \\
\hline Válido & Poco & 16 & $27 \%$ \\
& Regular & 16 & $27 \%$ \\
& Bastante & 28 & $47 \%$ \\
& Total & 60 & $100 \%$ \\
\hline
\end{tabular}

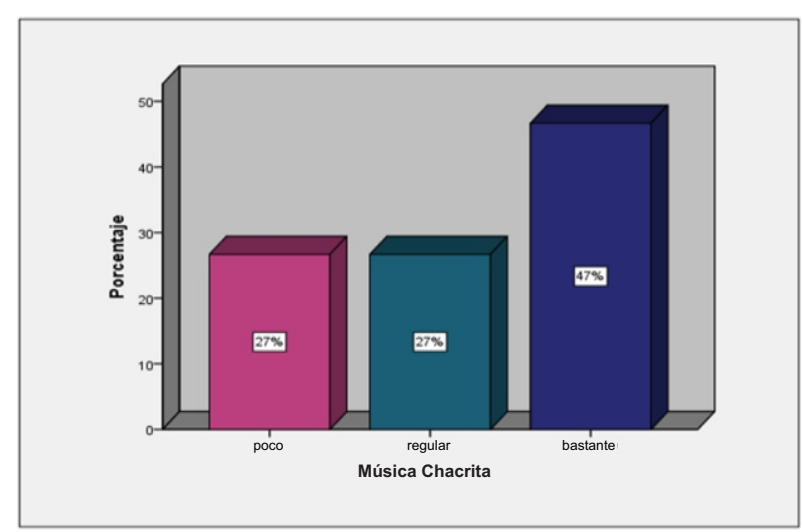

Figura 2. Gráfico de apreciación a la música chacrita por el poblador.
En la Tabla 2 y Figura 2, se observa que, 16 encuestados que representa el $27 \%$, manifiestan que, tienen poco apreciación a la música chacrita, de la misma manera 16 de los encuestados que representan $27 \%$ manifiestan que tienen apreciación regular, y 28 de los encuestados que representan al $47 \%$ manifiestan que su apreciación es bastante.

Tabla 4. Frecuencia y porcentaje de apreciación del pasacalle por el poblador.

\begin{tabular}{llcc}
\hline & & Frecuencia & Porcentaje \\
\hline Válido & Poco & 5 & $8 \%$ \\
& Regular & 39 & $65 \%$ \\
& Bastante & 16 & $27 \%$ \\
Total & 60 & $100 \%$ \\
\hline
\end{tabular}

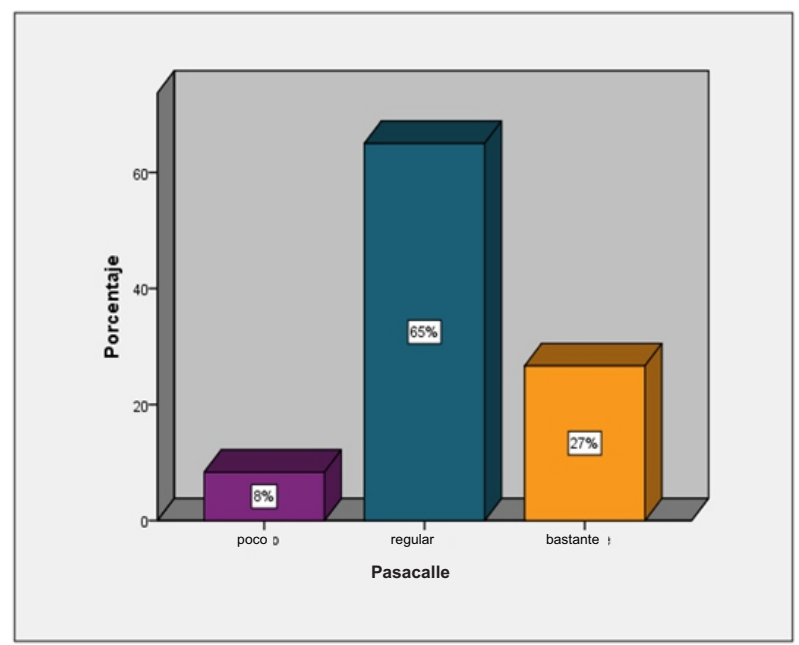

Figura 3. Gráfico de apreciación a la música pasacalle por el poblador.

En la Tabla 3 y Figura 3, se observa que, 5 encuestados que representa el $8 \%$, manifiestan que, tienen poco apreciación a la música pasacalle, de la misma manera 39 de los encuestados que representan el $65 \%$ manifiestan que tienen apreciación regular, y 16 de los encuestados que representan el $27 \%$ manifiestan que su apreciación es bastante.

Tabla 5. Frecuencia y porcentaje de apreciación del chimaychi por el poblador.

\begin{tabular}{llcc}
\hline & & Frecuencia & Porcentaje \\
\hline Válido & Poco & 18 & $30 \%$ \\
& Regular & 20 & $33 \%$ \\
& Bastante & 22 & $37 \%$ \\
Total & 60 & $100 \%$ \\
\hline
\end{tabular}




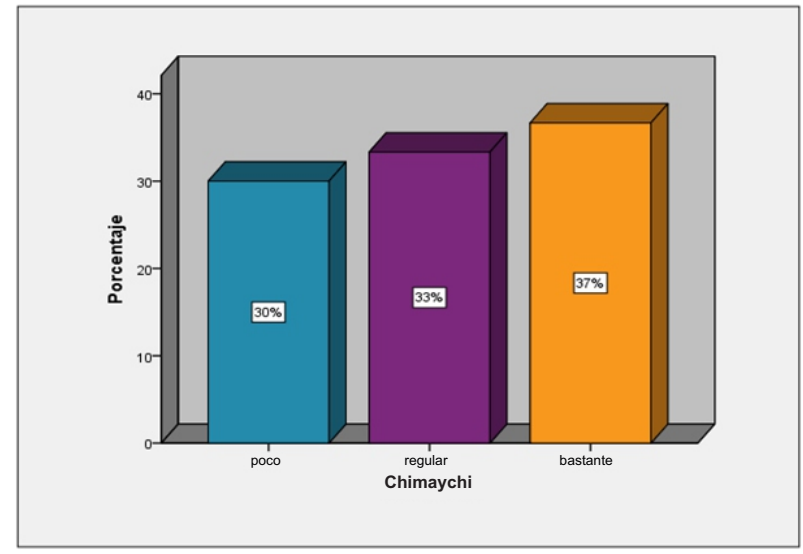

Figura 4. Gráfico de apreciación a la chimaychi por el poblador

De acuerdo a la Tabla 4 y Figura 4, se observa que, 18 encuestados que representa el $30 \%$, manifiestan que, tienen poco apreciación a la música chimaychi, de la misma manera 20 de los encuestados que representan el 33\% manifiestan que tienen apreciación regular, y 22 de los encuestados que representan el $37 \%$ manifiestan que su apreciación es bastante.

\section{DISCUSIÓN}

Arredondo (2006) efectuó la investigación titulada "dualidad simbólica de plantas y animales en la práctica médica del curanderopaciente en Huancayo" un estudio folclórico, cuyo objetivo fue Conocer la percepción de los pobladores de Huancayo en referencia a la dualidad simbólica, en el uso de plantas y animales como un hecho social en la práctica médica tradicional y en el sincretismo médico. Llegando a la conclusión de que existen evidencias folclóricas en que el curandero utiliza las plantas y los animales en formas duales, izándolos para realizar las curaciones y usando costumbres ancestrales de sus antepasados con rituales musicales.

Arévalo (2009) realizo la investigación titulada" Importancia del folklore musical como práctica educativa" fijando como objetivo el conocer la importancia del folklore musical como práctica educativa, arribando a las conclusiones como que la educación debe partir del entorno y el contexto del alumno, la música es esencial para poder realizar los repertorios populares y folclóricos que cada día se pierden, los estudiantes serán los aliados estratégicos para seguir trasmitiendo esta manifestación cultural, de generación en generación.
Iriarte (2009) mencionó que "el estudio del folklore musical, engloba la música tradicional viva". Esto indica que los diferentes pueblos vienen practicando la música folclórica, trasmitiendo a sus descendientes en forma oral, de generación en generación; y en la actualidad en algunos casos también se pueden trasmitir por vía académica, es decir haciendo uso del pentagrama. Esta manifestación de trasmisión directa da lugar que muchas personas que visitan el lugar aprecien las manifestaciones musicales. En muchas oportunidades les gusta la música, al regresar a sus lugares de origen y tratan de interpretar la manifestación, pero tienden a modificar la originalidad aunque sea en una pequeña parte. Como consecuencia se van gestando nuevas manifestaciones con esas pequeñas variaciones trasmitiéndose de pueblo en pueblo mediante vía oral.

El "huayno" como manifestación musical propicia la alegría en las reuniones de los diferentes pueblos, en forma musical o acompañado de cantantes. Su uso es frecuente por los pobladores para festejar cualquier actividad de índole cívica patriótica, institucional o como también una reunión familiar, en el que se aprecia realizar el baile en parejas entre un hombre y una mujer que bailan con un pañuelo en la mano derecha.

Debemos mencionar que dentro de los huaynos existe una división de acuerdo a su ejecución, por la manera de sus compas, diferenciándose entre ellos. Algunos son más rápidos en su ejecución, tal es el caso de la "chuscada", que es un baile picaresco, lleno de alegría y emoción en que la pareja zapatea a manera de contrapunto, gestándose la alegría y el aplauso de los asistentes que motivan bailar con más prestancia y elegancia tratando de ganar a la pareja con quien baila.

La música "chacrita", es un género musical que se diferencia de la chuscada por su manera de ejecutar los instrumentos en forma más pausada que esta con característica de melodía más gustosa especialmente la que proviene del violín, teniendo una diferencia con el ritmo del "chimaychi". Sin embargo, el "pasacalle" y los chimaychis son más pausados, pero cada uno de ellos con características propias. Lo más resaltante es que los pueblos del distrito de San Juan de Chullin, cultivan el chimaychi, desde muchos años atrás y/o van trasmitiendo en forma oral de 
generación en generación, perfeccionándose más y más, apareciendo músicos y cantantes nuevos en este género. De esta manera los pueblos del distrito de San Juan de Chullin son propietarios de esta manifestación por su práctica constante de muchos años atrás.

Al respecto Kapsoli, (2011) citó, "es folklórico lo creado directamente por el pueblo en forma anónima $y$, naturalmente, la que el mismo pueblo ha incorporado, también de manera anónima, de la música escrita". Estas prácticas de crear, les dan la facultad de ser sus propietarios, por consiguiente toda la manifestación que han creado dentro de la provincia resulta ser propiedad de sus pobladores, por ende de la provincia; y consideradas riquezas folclóricas del lugar.

Los diferentes pueblos de la tierra cultivan sus costumbres, sus creencias, y demás manifestaciones, dentro de ello la música, que cada pueblo practica de acuerdo a lo que la población hace con sus propias características, determinando una identidad propia del pueblo.

En la gran mayoría de jóvenes y adolescentes que viven en los pueblos de Sihuas, al entrevistarles se identifican y aprecian la música que se cultivan en sus pueblos.

Ruiz (2015) mencionó: "para tener conocimiento de qué papel juega la música en la construcción de una identidad, durante la adolescencia de manera fiel, cercana y realista, se debe realizar una encuesta". Necesariamente es recomendable hacer una encuesta a los participantes sobre sus preferencias y con cuál de los géneros musicales se identifican más. Algunas personas se dedican a formar grupos musicales, con los cuales practican constantemente identificándose con la población y tratan de difundir la música del lugar, tal es el caso de "los alegres de Chincho bamba" donde dos jóvenes músicos practican la música autóctona del lugar, que hoy en día es tan popular como es el chimaychi, manteniendo la originalidad, que le da una identidad de ser netamente del lugar como es el distrito de San Juan de Chullin.

Lo que nos permite entender que en el contexto psicosocial, la música desempeña un papel muy importante en la sociedad, por ser uno de los medios que permite a los adolescentes poder conocerse como tal, y verse reflejados con los demás. Para ello, procuraran buscar a otros de igual preferencia para identificarse formando un grupo, o estereotipos que practiquen los mismos géneros musicales. Al construir un grupo musical van a mantener la identidad musical y costumbres del lugar, gestándose dos situaciones como: elemento socializador y diferenciador.

Como elemento socializador, sirve para integrar a las personas mediante las reuniones disfrutando de la misma música, el tipo y género en común. Y por otro lado de diferenciador, para poder separar a las personas de acuerdo a la música que cada uno se identifica.

Los autores citados como Arévalo (2009), Arredondo (2006), Iriarte (2009) y Kapsoli (2011), consideran que el folclor musical de nuestros pueblos aun siendo variados, coinciden en el mensaje y su singular armonía propio de la manifestación nostálgica y otras veces alegre que trasmite el sentimiento popular por eso en la provincia de Sihuas, de las personas naturales de lugar que llegaban procedentes de los 10 distritos, sin tener preferencia de la edad ni el sexo de los encuestados, informándonos de la apreciación real del huayno, manifestación de gran trascendencia en la provincia. Teniendo en cuenta los cuatro géneros musicales que se practican en la provincia. Es así que se observa que el poblador Sihuasino tiene mayor preferencia por el género musical chuscada llegando a representar el $82 \%$ de los encuestados a nivel de la provincia, seguido por la música chacrita con un $47 \%$, seguido por el chimaychi con un $37 \%$ de apreciación. El género pasacalle se ubica en un $27 \%$ de preferencia.

Finalmente, quedando abierto el tema, para las futuras investigaciones que se pretendan realizar referente al caso, pudiendo tomar dos o más variables para poder tener información más profunda, que ayuden solucionar 0 plantear nuevas investigaciones que nos lleven a nuevos resultados. Además se puede realizar investigaciones con mayor muestra considerando el ámbito de mayor extensión organizando la muestra por estratos por cada distrito. Otro punto de mayor trascendencia se realizaría teniendo en cuenta el bilingüismo en los distritos de San Juan de Chullín, Sicsibamba, una parte del distrito de Huayllabamba, cuyos resultados servirían a los educadores para tener en cuenta plantear una 
educación bilingüe, a fin de adecuar los aprendizajes al idioma que les facilitaría el aprendizaje con mayor interés y con provecho a los estudiantes de esos pueblos.

\section{REFERENCIAS BIBLIOGRÁFICAS}

Arredondo Baquerizo, F. L. (2006). Dualidad simbólica de plantas y animales en la práctica médica del curandero-paciente en Huancayo. Tesis para optar el Grado de Magister, Pontificia Universidad Católica del Perú, Lima.

Arévalo Galán, A. (2009). Importancia del folklore musical como práctica educativa. Revista electrónica de LEEME, (23). Recuperado el 9 de F e b r e r o d e 2017 d e http://musica.rediris.es/leeme ISSN: 1575-9563.
Kapsoli, W. (1986). Nosotros los maestros. Ensayos sobre la educación peruana, 239-258.

Iriarte, F. (2009). Cuaderno Arguedianas No $9-$ Escuela Nacional Superior de Folklore Josè Marìa Arguedas, Editorial Turis América Editores Gráficos.

Ruiz, A. (2015). El papel de la música en la construcción de una identidad durante la adolescencia, ¿Dime qué escuchas y te diré quién eres? Revista de musicología Sinéresis (22).

\section{Correo electrónico:}

diestrasalinas@hotmail.com

Revisión de pares:

Recibido: 02-05-2017

Aceptado:20-06-2017 